\title{
Monopole condensation in the ground state of gauge theories: a disorder parameter.
}

\author{
L. Del Debbio, A. Di Giacomo and G. Paffuti * a

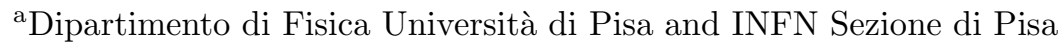

We construct a disorder parameter for dual superconductivity of the ground state of $U(1)$ gauge theory.

\section{Introduction}

The main motivation of this work is understanding the mechanism of colour confinement in QCD. An appealing possibility is dual superconductivity of the ground state 1 . that the role of electric and magnetic quantities is interchanged with respect to ordinary superconductors: the chromoelectric field acting between a $q \bar{q}$ pair is then channeled by dual Meissner effect into Abrikosov flux tubes, which behave as strings [4]. Such tubes have been observed in lattice configurations [7]. The question is: can we directly detect dual superconductivity on the lattice? The problem is always reduced to a $U(1)$ problem by the so called abelian projection [1,89,9]: our task is then to characterize dual superconductivity of a $U(1)$ system, and to find an unambigous way to detect it.

Dual superconductivity is the spontaneous breaking à la Higgs of the $U(1)$ symmetry related to the conservation of magnetic charge: monopole charges condense in the vacuum (in the same way as Cooper pairs do in ordinary superconductors), making it $U(1)$ non invariant [10]. If the vacuum is $U(1)$ invariant the vacuum expectation value $(v e v)$ of any operator with non zero magnetic charge is zero. Spontaneous breaking of $U(1)$ is therefore signalled by non vanishing vev of any magnetically charged operator. Such vev is called a disorder parameter in the language of statistical mechanics 11. A rigorous proof exists 12 that in compact $U(1)$ on the lattice monopoles do condense for $\beta=1 / e^{2}$ smaller than some crit-

\footnotetext{
*Partially supported by MURST and by EC Contract
} CHEX-CT92-0051 ical value $\beta_{c}$ : the proof requires a specific form of the action (Villain) and infinite volume, to perform the transformation to dual variables 13 . The difficulties with finite volume were already noticed in ref 11 for the Ising model. We have constructed a disorder parameter which coincides with that of ref 12 for Villain action and infinite volume, but can be used with any form of the action and can detect dual superconductivity in finite lattices.

\section{Construction of the disorder parameter}

As magnetically charged operator we shall use a creation operator for monopoles, defined as follows 14. Let $\Pi_{i}(\mathbf{x}, t)=F_{0 i}(\mathbf{x}, t)$ be the usual conjugate momentum to the field variables $A_{i}(\mathbf{x}, t)$. Then the operator

$\mu(\mathbf{y}, t)=\exp \left[\mathrm{i} \int \mathrm{d}^{3} \mathbf{x} \Pi_{i}(\mathbf{x}, t) \frac{1}{e} b_{i}(\mathbf{x}-\mathbf{y})\right]$

creates a monopole if $b_{i}(\mathbf{x}-\mathbf{y})$ is the vector potential produced in $\mathbf{x}$ by a monopole of charge $\mathrm{m} / \mathrm{e}$ sitting in $\mathbf{y}$. Putting the string along the 3 axis, and $\mathbf{r}=\mathbf{x}-\mathbf{y}$,

$b_{i}(\mathbf{x}-\mathbf{y})=\frac{m}{2} \frac{\varepsilon_{3 i j} r_{j}}{r\left(r-r_{3}\right)}$

In fact $\mu$ is a translation operator of the field, as is easily seen in the Schrödinger picture

$\mu(\mathbf{y}, t)\left|A_{i}(\mathbf{x}, t)\right\rangle=\left|A_{i}(\mathbf{x}, t)+b_{i}(\mathbf{x}, \mathbf{y})\right\rangle$

In the same language if $\nabla \wedge \mathrm{g}=0$ the operator

$\gamma(t)=\exp \left[\frac{\mathrm{i}}{e} \int \mathrm{d}^{3} \mathbf{x} \Pi_{i}(\mathbf{x}, t) g_{i}(\mathbf{x})\right]$ 
defines a gauge transformation

$\gamma(t)\left|A_{i}(\mathbf{x}, t)\right\rangle=\left|A_{i}(\mathbf{x}, t)+g_{i}(\mathbf{x})\right\rangle$

The correct prescription to define vev of $\mu$ in the euclidean region with Feynman path integral can be checked on a system of free photons, with periodic boundary conditions. It amounts to define the vev of the operator $\mu$ as 14]

$\langle\bar{\mu}\rangle=\frac{\left\langle\mu\left(\mathbf{y}, y_{4}\right)\right\rangle}{\left\langle\gamma\left(y_{4}\right)\right\rangle}=\frac{\int \mathcal{D} A_{\mu} \exp \left[-\beta\left(S+S_{b}\right)\right]}{\int \mathcal{D} A_{\mu} \exp \left[-\beta\left(S+S_{g}\right)\right]}$

where

$$
\begin{aligned}
S_{b} & =\int \mathrm{d}^{3} \mathbf{x} F_{4 i}\left(\mathbf{x}, y_{4}\right) b_{i}(\mathbf{x}-\mathbf{y}) \\
S_{g} & =\int \mathrm{d}^{3} \mathbf{x} F_{4 i}(\mathbf{x}) g_{i}(\mathbf{x})
\end{aligned}
$$

$S$ is the action and $g_{i}$ is any gauge configuration subjected to the condition that

$$
\int \mathrm{d}^{4} k|\tilde{\mathbf{b}}(k)|^{2}=\int \mathrm{d}^{4} k|\tilde{\mathbf{g}}(k)|^{2}
$$

The factor $\beta$ in the second term of the exponents come from the factor $1 / e$ in the magnetic charge of the monopole times the $1 / e$ coming from the usual rescaling of the fields, the same which produces the factor $\beta$ in front of $S$. For free photons (or for $\beta>\beta_{c}$ ) $S=F_{\mu \nu} F^{\mu \nu} / 4$, the integral (5) is gaussian and

$$
\begin{aligned}
\langle\bar{\mu}\rangle & =\exp \left[-\frac{\beta}{4} \int \frac{\mathrm{d}^{3} \mathbf{k}}{(2 \pi)^{3}}|\mathbf{k}||\tilde{\mathbf{b}}(\mathbf{k})|^{2}\right] \\
& =C \exp \left[-\beta \ln \left(\frac{V}{a^{3}}\right)\right]
\end{aligned}
$$

$a$ is some ultraviolet cutoff. $\langle\bar{\mu}\rangle \rightarrow 0$ as $a \rightarrow 0$ or at fixed $a$ (e.g. on a lattice) as $V \rightarrow \infty$ on the lattice. Our disorder parameter becomes an order parameter of the dual theory, i.e. exactly zero for $\beta>\beta_{c}$, only in the limit $V \rightarrow \infty$ : with a finite number of degrees of freedom, being analytic in $\beta$, it cannot be zero for $\beta>\beta_{c}$, since then it would be identically zero 11. What is really important, however, in order to detect superconductivity, is to show that it is different from zero below $\beta_{c}$, independent of the finiteness of the volume. To accomplish this we will compute $\langle\bar{\mu}\rangle$ not directly, but through

$$
\begin{aligned}
\rho_{b}(\beta) & =\frac{\mathrm{d} \ln \langle\bar{\mu}\rangle}{\mathrm{d} \beta}= \\
& =\left\langle S+S_{g}\right\rangle_{S+S_{g}}-\left\langle S+S_{b}\right\rangle_{S+S_{b}}
\end{aligned}
$$

The label on the right of the brackets indicates the action used to compute the average: eq.(7) directly follows from eq.(5). Since $\langle\bar{\mu}\rangle_{\beta=0}=1$, in terms of $\rho_{b}$

$\langle\bar{\mu}\rangle=\exp \left(\int_{0}^{\beta} \rho_{b}\left(\beta^{\prime}\right) \mathrm{d} \beta^{\prime}\right)$

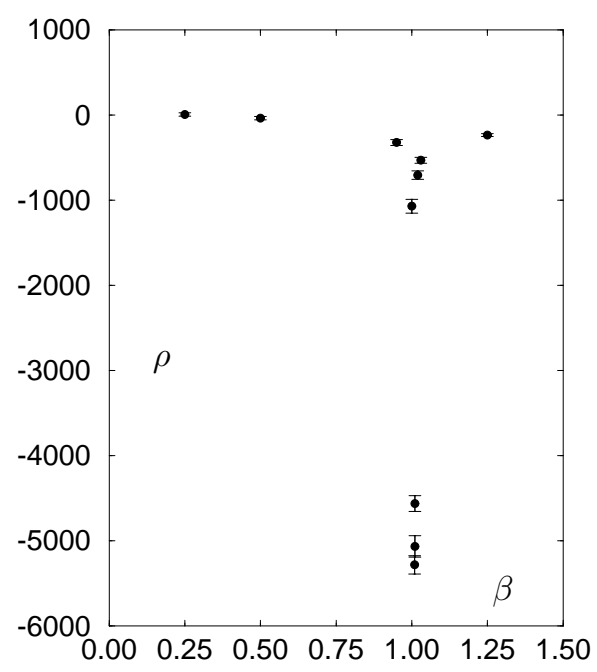

Fig. 1: $\rho_{b}(\beta)$ on a $12^{4}$ lattice.

Computing $\langle\bar{\mu}\rangle$ from $\rho$ has a few advantages.

(i) It solves the problem of fluctuations produced by the fact that $\langle\bar{\mu}\rangle$, being the average of the exponent of an extensive quantity, has a non gaussian distribution 15.

(ii) It eliminates problems of boundary conditions in the definition of $\langle\bar{\mu}\rangle 13$. Indeed the r.h.s. of eq.(7) is the average of an extensive quantity, and is sensitive to the bulk properties of the system at least for $\beta<\beta_{c}$, 
i.e. in the presence of a mass gap. Computing $\langle\bar{\mu}\rangle$ with periodic and antiperiodic b.c. gives the same result within errors.

To be rigorous we should have started from the correlation function betwen a monopole - antimonopole pair, defining $\langle\bar{\mu}\rangle$ through the cluster property

$\frac{\langle\mu(x) \mu(y)\rangle}{\left\langle\gamma^{2}\right\rangle} \underset{|x-y| \rightarrow \infty}{\simeq}\langle\bar{\mu}\rangle^{2}$

Putting $S_{b \bar{b}}=S_{b}+S_{\bar{b}}$

$$
\begin{aligned}
\rho_{b \bar{b}} & =\frac{\mathrm{d}}{\mathrm{d} \beta} \ln \frac{\langle\mu(x) \mu(y)\rangle}{\left\langle\gamma^{2}\right\rangle}= \\
& =2\left\langle S+S_{g}\right\rangle_{S+S_{g}}-\left\langle S+S_{b \bar{b}}\right\rangle_{S+S_{b \bar{b}}}-\langle S\rangle_{S}
\end{aligned}
$$

the cluster property reads then, for $|x-y| \rightarrow \infty$

$\rho_{b \bar{b}}=2 \rho_{b}$

However if the correlation length is much smaller than the lattice size, measuring $\rho_{b \bar{b}}(\beta)$ or $\rho_{b}(\beta)$ are equivalent procedures.

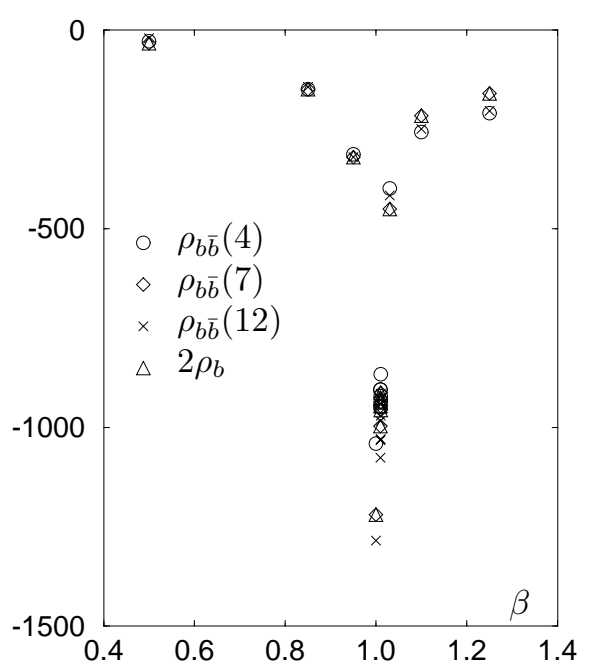

Fig. $2: \rho_{b \bar{b}}(\beta)$ and $2 \rho_{b}(\beta)$ on a $6^{3} \times 20$ latice.

Numerical results on a $6^{3} \times 20$ for $\rho_{b}$ and $\rho_{b \bar{b}}$ are shown in fig.2, and confirm the validity of the cluster property at distances $d \geq 4$. $\rho_{b}$ for a lattice $12^{4}$ is shown in fig.1.
At large $\beta$ 's $\rho$ agrees with eq.(6). A large negative peak at $\beta=\beta_{c}$, whose area is an increasing function of $V$, signals a vanishing $\langle\bar{\mu}\rangle$. For $\beta<\beta_{c}$ $\langle\bar{\mu}\rangle$ becomes volume independent at sufficiently large volumes, showing unambigously monopole condensation.

In conclusion we have a reliable disorder parameter which can be used with any form of the action, at finite volume, to detect dual superconductivity of the vacuum.

\section{REFERENCES}

1. G. 't Hooft, in "High Energy Physics", EPS International Conference, Palermo 1975, ed. A. Zichichi; Nucl Phys. B 190 (1981) 455.

2. S. Mandelstam, Phys. Rep. 23C (1976) 245.

3. G. Parisi, Phys. Rev. D 11 (1975) 970.

4. G. Veneziano, Nuovo Cimento 57 A (1968) 190.

5. H.B. Nielsen, P. Olesen, Nucl. Phys. B61 (1973) 45.

6. Y. Nambu, Phys. Rev. D 10 (1974) 4262.

7. A. Di Giacomo, M. Maggiore and S. Olejník, Phys. Lett. B236 (1990) 199; Nucl. Phys. B347 (1990) 441; R.W. Haymaker, J. Wosiek, Phys. Rev. D 36 (1987) 3297;

8. A.S. Kronfeld, G. Schierholz, U.J. Wiese, Nucl. Phys. B293(1987) 461.

9. L. Del Debbio, A. Di Giacomo, G. Paffuti, G. Pieri, these proceedings.

10. S. Weinberg, Progr. of Theor. Phys. Suppl. No. 86 (1986) 43.

11. L.P. Kadanoff, H.Ceva, Phys. Rev. B3 (1971) 3918.

12. J. Frölich, P.A. Marchetti, Comm. Math. Phys. 112 (1987) 343.

13. L. Polley, U. Wiese, Nucl. Phys. B 356 (1991) 629.

14. L. Del Debbio, A. Di Giacomo, G. Paffuti, IFUP-TH- 16/94; submitted for publication.

15. A. Hasenfratz, P. Hasenfratz, F. Niedermayer, Nucl. Phys. B 239 (1990) 739. 